\title{
O SENSORIAMENTO REMOTO APLICADO A UM MODELO DE CARTAS DE PESCA
}

\author{
SYDNÉA MALUF \\ Instituto de Pesquisas Espaciais - INPE \\ São José dos Campos, SP - Brasil
}

\begin{abstract}
SYNOPSIS
A methodology for the determination of the best potential fishing zones, for sardine, in the Brazilian coastal area of the Southwestern Tropical Atlantic Ocean, between latitudes $21^{\circ} 45^{\prime} \mathrm{S}$ and $25^{\circ} 00^{\prime} \mathrm{S}$ and longitudes $40^{\circ} 50^{\prime} \mathrm{W}$ and $47000^{\prime} \mathrm{W}$ is presented. By using this methodology, a fishing chart model is developed from observations relative to the months of July, August, September, November and December. Ma. rine fishing chart containing such zones are presented for the September month. The potentiality of the VHRR.IR and the surface charts of NOAA's satellite, as a help in the determination of the fishing zones is also demonstrated.
\end{abstract}

Introdução

Com o lançamento do satélite terrestre LANDSAT-1 (ini cialmente ERTS-1 - "Earth Resources 1echnology Satellite 1") pela NASA ("National Aeronautics and Space Administration"), em Julho de 1972, pesquisadores ligados à pesca voltaram a atenção para o uso de suas potencialidades, no levantamento e monitoramento dos recursos pesqueiros. As pesquisas inicialmente realizadas, com o satélite LANDSAT-1, combinavam o uso de embarcaçres pesqueiras e pilotos de reconhecimento (spotter pilot), operando dentro ou perto das áreas de estudo. A finalidade era verificar as correlações existentes entre os dados do MSS (Multispectral Scanner) do LANDSAT-1, os padrões de distribuição do peixe e alguns parâmetros oceanográficos, tais como: cor do oceano, teor de clorofila, turbidez da água, temperatura e salinidade. Alguns resultados dessas correlações são encontrados em Kemmerer \& Benigno (1973), Maughan, Marmelstein \& Temple (1973), Bullis \& Kemmerer (1976), Kemmerer \& Butler (1977).

Assim como o LANDSAT, os satélites meteorológicos comȩaram a ser utilizados para levantamento de recursos pesqueiros, como por exemplo, os satélites da série NOAA ("National Oceanic and Atmospheric Administration"). Oceanógrafos da IATTC ("Inter American Tropical Tuna Commission") demonstraram a viabilidade do uso das imagens infravermelho termais (IR), do VHRR (Very High Resolution Radiometer) no monitoramento de áreas do oceano com temperaturas superficiais mais quentes ou mais frias que a normal, assim como das frentes oceânicas, tão importantes à pesca (Stevenson \& Miller, 1973; 1974 a e b). Stevenson, Miller \& Kirkham (1975 e 1976) e Stevenson \& Kirkham (1976) demonstraram a utilidade do VHRR (NOAA) e do MSS (LANDSAT) no campo da oceanografia pesqueira. Através dos dados infravermelho, visual e colorido, coletados desses dois satélites, os autores indicam as aplicações do conjunto dessas informações, no monitoramento das condiçōes da superfície do mar, no Pacífico Norte.

Este trabalho apresenta uma metodologia desenvolvida no INPE, aproveitando, evidentemente, a experiência existente.

\section{Metodologia}

Foram elaboradas cerca de trinta e uma cartas de distribuição superficial de temperatura $\left({ }^{\circ} \mathrm{C}\right)$, salinidade $(\% \circ)$, oxigênio $(m \ell / \ell)$, fosfato $(\mu \mathrm{g}$ at $/ \ell$ ), vento (graus e nós) e de captura de sardina ( $\mathrm{kg} /$ lance), para os seis meses estudados (julho a dezembro). Essas cartas foram confeccionadas a partir de valores médios mensais, locados em quadrados de $0,5^{\circ} \times 0,5^{\circ}$ e $1^{\circ} \times 1^{\circ}$ (no caso especial das Cartas de Vento), com base na carta n. 23900 da Diretoria de Hidrografia e Navegação (DHN), Ministério da Marinha (escala natural 1:1.000.000).

Foram subdivididas, as ditas cartas, em: Cartas Básicas n? 1 , 2,3 e 4 segundo a etapa da sua elaboração e utilização e denominadas Básicas porque contém as informações básicas e essenciais à delimitação das zonas mais propícias à pesca (Inostroza \&Maluf, 1978; Mahuf, 1978).

Tendo-se determinado as zonas mais propícias à pesca, em função do índice de captura de sardinha ( $\mathrm{kg} /$ lance) e dos parâmetros oceanograficos a ela associados, estas foram transportadas para uma outra série de cartas, consideradas segundo o Modelo aqui desenvolvido, as Cartas de Pesca propriamente ditas. Essas zonas ficaram ilustradas como núcleos distintos, distribuídos ao longo de toda a costa da área em estudo. Determinou-se para cada mês:
- o número total de zonas mais propícias à pesca contidas na respectiva Carta de Pesca;

- suas localizações: pelas coordenadas geográficas aproximadas do centro de cada zona mais propícia à pesca, pela distância da costa ao limite mais próximo da zona analisada (em quilômetros e milhas náuticas) e pela profundidade local;

- a dimensão $(\mathrm{km})^{2}$ de cada zona;

- o valor aproximado em $\mathrm{kg} / \mathrm{lance}$ de sardinha capturada.

Foi realizada uma comparação qualitativa (visual), entre a imagem do VHRR infravermelho termal, do satélite NOAA-5, correspondente ao dia 29 de setembro de 1977 (órbita no 5282, horário $10: 43 \mathrm{Z}$ ). e a carta oceanográfica de temperatura sunerficial, obtida com os valores médios referentes ao mes de setembro. A área de estudó, contida nessa imagem, foi ampliada e os contrastes térmicos apresentados na água do mar, realçados segundo a técnica descrita por Corbell et al. (1976), no laboratório fotográfico do INPE em São José dos Campos. Sobre essa imagem foi feita uma transparência. delimitando as áreas mais claras e escuras da parte correspondente ao mar (respectivamente regiōes de águas frias e quentes). Nessa transparência foram ilustradas, também, as zonas mais propícias à pesca, contidas na Carta de Pesca do mês de setembro.

Posteriormente, fez-se uma comparação das cartas contendo as isotermas traçadas a partir dos dados de temperatura de superfície (obtidas pelo satélite NOAA-4), com a mesma carta oceanográfica de temperatura superficial, utilizada anteriormente (mês de setembro). As informações sobre a temperatura da superfície do mar, obtidas pelo satélite NOAA-4, assim como as isotermas traçadas a partir desses dados, correspondem às imagens dos dias 3 de setembro de 1975 e 7 de setembro de 1976 e representam a temperatura de superfície do mar, para a área compreendida entre as latitudes de $15^{\circ} 00^{\prime} \mathrm{S}$ e $30^{\circ} 00^{\prime} \mathrm{S}$ e longitudes de $034^{\circ} 00^{\prime} \mathrm{W}$ e $049^{\circ} 00^{\prime} \mathrm{W}$. Maiores detalhes poderão ser encontrados em Ikeda, 1977.

Sobre essa área, delineamos a região correspondente à área de estudo deste trabalho e, dentro desta, em asterisco, representaram-se as zonas mais propícias à pesca do mês de setembro (guardadas as devidas proporções de escala). Para exemplificar, foram consideradas somente duas das zonas mais propícias à pesca: a que se localizou frente à Baía de Guanabara (zona 1) e a situada entre Iltha Grande e Ilha de São Sebastião (zona 2).

\section{Resultados}

Os resultados alcançados dizem respeito somente a cinco dos seis meses estudados: julho, agosto, setembro, novembro e dezembro. No mês de outubro, a distribuição da captura de sardinha ocorreu até a isóbata de 150 metros (entre Cabo de São Tomé e Baía de Guanabara) sendo que, frente à Ilha de São Sebastião, ocorreu entre as isóbatas de 50 a 100 metros. Analisando, por outro lado, as cartas oceanográficas desse mês, pôde-se constatar que a distribuição dos parâmetros ocorreu mais além da costa, pouco ou nada coincidindo com a distribuição da captura de sardinha, sendo portanto, impossível a correlação entre essa e os parâmetros ambientais.

A Figura 1 ilustra uma das cinco cartas de Pesca, relativa ao mês de setembro, contendo as zonas mais propícias à pesca, determinadas a partir da metodologia estabelecida nesse primeiro modelo de Carta de Pesca.

A Tabela 1 indica, para esse mesmo mês, o número de zonas mais propícias à pesca e suas características. Essas zonas encon- 


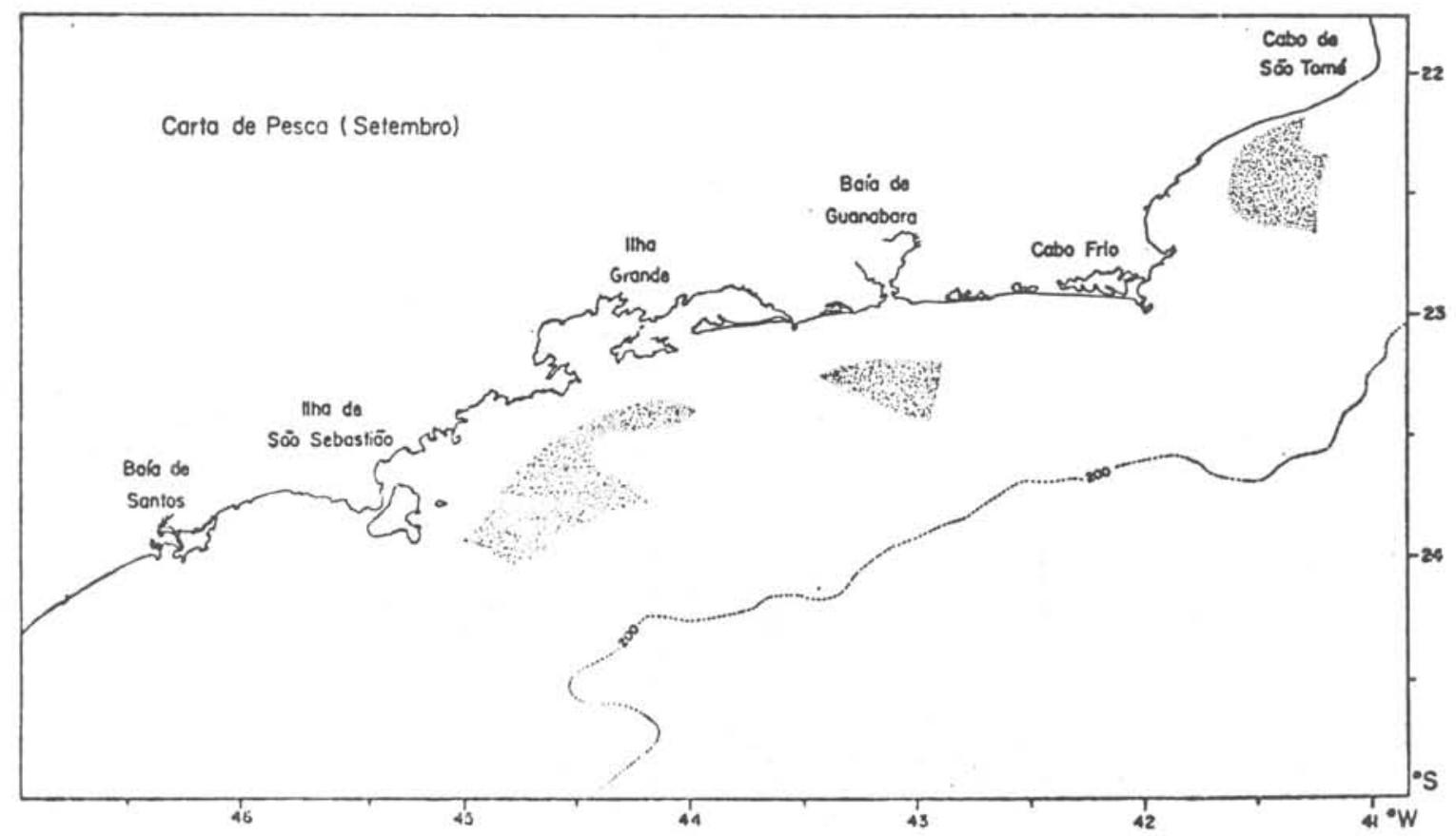

Fig. 1 - Carta de Pesca em Setembro,

TABELA I

INFORMAÇÕES SOBRE AS ZONAS MAIS PROPICIAS À PESCA

DA CARTA DE PESCA DO MÊS DE SETEMBRO

\begin{tabular}{|c|c|c|c|c|c|c|c|}
\hline \multirow{3}{*}{ ZONA } & \multicolumn{5}{|c|}{ LOCALIZAÇĀO } & \multirow{3}{*}{$\begin{array}{l}\text { INDICE DE } \\
\text { CAPTURA }\end{array}$} & \multirow{3}{*}{$\begin{array}{c}\text { DIMENSĀO } \\
\left(\mathrm{km}^{2}\right)\end{array}$} \\
\hline & \multicolumn{2}{|c|}{ COORDENADAS DE CENTRO } & \multicolumn{2}{|c|}{$\begin{array}{l}\text { DISTÂNCIA APROXI- } \\
\text { MADA DA COSTA }\end{array}$} & \multirow{2}{*}{$\begin{array}{c}\text { PROFUN- } \\
\text { DIDADE } \\
\text { LOCAL } \\
\text { (m) }\end{array}$} & & \\
\hline & Latitude & Longitude & $(\mathrm{km})$ & $(\mathrm{nm})$ & & & \\
\hline & & - & & & & & \\
\hline 1 & $22^{\circ} 25^{\prime} \mathrm{S}$ & $41^{\circ} 25^{\prime} \mathrm{W}$ & 9 & 5 & $10-60$ & $>7.000$ & 1.669 \\
\hline 2 & $23^{\circ} 20^{\prime} \mathrm{S}$ & $43^{\circ} 10^{\prime} \mathrm{W}$ & 28 & 15 & $90-120$ & $>14.000$ & 1.027 \\
\hline 3 & $23^{\circ} 45^{\prime} \mathrm{S}$ & $44^{\circ} 35^{\prime} \mathrm{W}$ & 28 & 15 & $50-100$ & $>14.000$ & 2.910 \\
\hline \multicolumn{7}{|c|}{ Total de área mais propícia à pesca } & 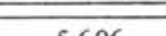 \\
\hline
\end{tabular}

tram-se numeradas nas tabelas, para facilitar sua identificação, devendo ser consideradas, na carta, da direita para a esquerda. Observa-se pela figura, que foram três as zonas mais propícias à pesca determinadas para esse mês: a primeira entre Cabo de São Tomé e Cabo Frio, definida pelos parâmetros: temperatura e salinidade, com intervalos entre $20^{\circ} \mathrm{C}$ a $21^{\circ} \mathrm{C}$ e $36,00 \%$ a $36,10 \%$; a segunda, localizada frente à Baía de Guanabara, foi definida pelos parâmetros temperatura, salinidade, oxigênio e fosfato com valores compreendidos entre $20^{\circ} \mathrm{C}$ e $21^{\circ} \mathrm{C} .35 .50 \%$ a $36.20 \%$ 。 $5,0 \mathrm{~m} / / \ell$ a $5,2 \mathrm{~m} \ell / \ell$ a $0,30 \mu \mathrm{g}$ at $/ \ell$ a $0,35 \mu \mathrm{g}$ at $/ \ell$ respectivamente; terceira e última zona mais propícia à pesca localizou-se entre a llha Grande e Ilha de São Sebastião, definida também pelos quatro parâmetros oceanográficos acima mencionados, com valores compreendidos entre $22^{\circ} \mathrm{C}$ a $23^{\circ} \mathrm{C}, 35,10 \%$ a $36,00 \% \circ 4,8 \mathrm{ml} / \mathrm{l}$ a $5,0 \mathrm{~m} \ell / \ell$ e $0.30 \mu \mathrm{g}$ at $/ \ell$ a $0.40 \mu \mathrm{g}$ at $/ \ell$ respectivamente.

Pelos resultados obtidos, constatou-se que a zona mais propicia à pesca da sardinha, mais distante da costa, foi a do més de julho, localizada a aproximadamente $37 \mathrm{~km}$ da costa $(20 \mathrm{mn})$, entre Cabo de São Tomé e Cabo Frio. Nos meses de setembro, novembro e dezembro, observaram-se zonas mais próximas à costa (cerca de $9 \mathrm{~km}$ de distância, ou $5 \mathrm{mn}$ ). Registrou-se para o més de setembro, a maior área correspondente às zonas mais propícias à pesca, com um total de $5606 \mathrm{~km}^{2}$ (Tabela I) e a menor, a do mes de novembro, com $3551 \mathrm{~km}^{2}$. A zona mais propícia à pesca lo calizada sobre a região de maior profundidade foi a do mês de setembro, situada entre Ilha Grande e Ilha de São Sebastião entre 90 e 120 metros (Figura 1); a localizada sobre a regiāo de menor profundidade foi a do mês de novembro, situada entre 10 e 40 metros (entre Cabo de São Tomé e Cabo Frio).

Comparando a imagem termal do satélite NOAA-5 com a carta oceanográfica de temperatura superficial, constatou-se que as áreas mais claras da imagem (água mais fria), corresponderam a regiões de temperatura menor que $22^{\circ} \mathrm{C}$ na carta oceanográfica, e as áreas mais escuras (água mais quente) corresponderam a temperaturas maiores que $22^{\circ} \mathrm{C}$, na carta oceanográfica. Com relação à localização das zonas mais propícias à pesca no mês de setembro, observou-se que as zonas 1 e 2 (localizadas respectivamente entre Cabo de São Tomé e Cabo Frio e frente à Baía de Guanabara) situaram-se numa regiāo de baixa temperatura (áreas claras). Essas zonas foram definidas por um mínimo de temperatura (menor que $21^{\circ} \mathrm{C}$ ). A terceira zona, localizada entre Illha Grande e llha de São Sebastiâo, está sobre uma região de águas mais quentes, na ima gem do NOAA-5, e foi definida por um máximo de temperatura (entre $22^{\circ}$ e $23^{\circ} \mathrm{C}$ ). 
A Figura 2 indica as isotermas traçadas a partir dos dados de temperatura da superfície do mar, obtida através do satélite NOAA-4, do dia 3 de setembro de 1975 . Comparando essa carta com a carta oceanográfica de temperatura superficial observou-se que a zona 1 localizou-se numa região onde a temperatura, fornecida pelo NOAA-4 foi de $20^{\circ} \mathrm{C}$ e a temperatura dada pela carta oceanográfica foi $21^{\circ} \mathrm{C}$; na região da zona 2 , a temperatura do NOAA-4 foi $21^{\circ} \mathrm{C}$ e a oceanográfica ficou entre $22^{\circ}$ e $23^{\circ} \mathrm{C}$. Verifica-se, portanto, que a diferença entre a temperatura registrada pela carta do satélite NOAA-4 e a da carta oceanográfica, nas regiōes: da zona 1 e 2 , foi de $\pm 1^{\circ} \mathrm{C}$. Smith et al (1970), obtiveram os mesmos resultados, utilizando imagens do THIR (Temperature Humidity Infrared Radiometer) do satélite NIMBUS.

\section{Conchusão}

Esse primeiro Modelo de Cartas de Pesca apresentou um número de zonas propícias à pesca diferente a cada mês, assim como suas localizações e os valores dos parâmetros oceanográficos que a definiram. Isso é esperado, principalmente quanao se considera fenômenos como migrações, variações ambientais, etc., que ocorrem de um mês para o outro numa mesma região. Esses fatores são importantes e precisam ser levados em consideração quando se pretende avaliar, não somente o número de zonas mais propícias à pesca mas, também, suas localizações. Pela comparação das informações oceanográficas com as de sensores remotos, pôde-se concluir a utilidade dos dados de temperatura, obtidos através dos satélites da série NOAA; no auxílio à determinação das zonas acima referidas. As cartas de temperatura da superfície do mar, obtidas através de dados dos satélites meteorológicos podem ser consideradas cartas básicas no levantamento e monitoramento dos recursos pesqueiros.

\section{Bibliografia}

BULLIS, H.R.J. \& KEMMERER, A.J. 1976. Examples of aerospace remote sensing applications to fisheries investigations.

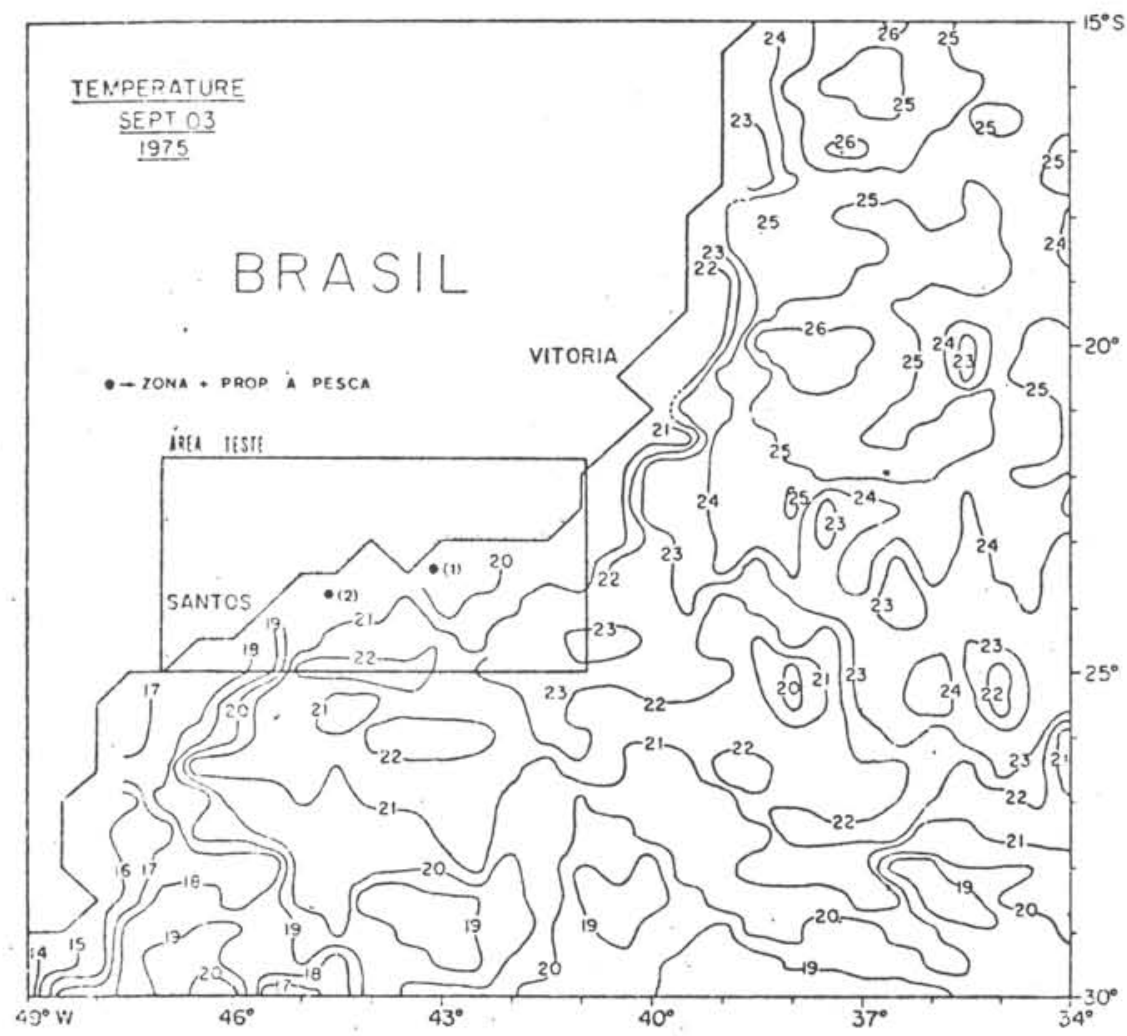

Fig. 2 - Isotermas obtidas dos dados de temperatura da superfície do mar (Satélite NOAA-4), correspondente ao dia 3 de setembro de 1975 (segundo Ikeda \& Pereira $F^{0 . ., 1978) . ~}$

Lenmark, International Council for Exploration of the Sea.

CORBELL, R.P.; CALLAHAM, C.J. \& KOTSCH, WJ.1976. Operational enhancement curves in active memory bank. The GOES/SMS user's guide. Washington, D.C., NOAA/NESS, p. 75 .

IKEDA, Y. 1977. Influências sazonais nas propriedades oceanográficas em grande, média e pequena escalas, de Cabo Frio-RJ a Cananéia-SP, baseadas nos dados obtidos pelo N/Oc. "Prof. W. Besnard" e satélite NOAA-4. Tese de doutorado. Universidade de São Paulo, Instituto Oceanográfico.

INOSTROZA, V. \& MALUF, S. 1978. A tlas de estruturas oceanográficas da costa sudeste do Brasil para estudos de sensoriamento remoto. São José dos Campos, INPE-1179-NTE/108, v. 1 e 2 .

KEMMERER, A. J. \&BENIGNO, J. A. 1973. Relationships between remotely sensed fisheries distribution information and selected oceanographic parameters in the Mississipi Sound. Symposium on Significant Results obtained from the Earth Resources Technology Satellite-1. Maryland, v. 1, p. $1685-1695$.

Mar. Fish. Rev., 39 (1) : 16-21.

MALUF, S. 1978. Cartas de pesca: um modelo de cartas de pesca utilizando dados oceanográficos e de sensoriamento , moto aplicado à sardinha. Tese de Mestrado. São José dos Campos, SP, Instituto de Pesquisas Espaciais.

MAUGHAN. P. M.; MAR MELSTEIN, A. S \&TEMPLE, O. R. 1973. Application of ERTS-1 imagery to the harvest model of the menhaden fishery. Symposium on Significant Results obtained from the Earth Resources Technology Satellite-1. Maryland, v. 1, p.1405-1411.

SMITH, W. L. RAO, P. K.; KOFFLER, R. \&CURTIS, W. R. 1970. The determination of sea-surface temperature satellite high resolution infrared window radiation measurements. Mon Weath. Rev. U.S. Dep. Agric., 98 (8) : 604-611.

STEVENSON, M. R. \& KIRKHAN, R. G. 1976. On the use of satellite scanning radiometer data to monitor sea surface temperatures. III Simp. latinoamer. Oceanogr. biol., San Salvador.

- - \& MILLER, F.R. 1973. Application of high resolution infrared and visual data to investigate changes in and the relationship between sea surface temperature and cloud patterns over the eastern tropical Pacific. La Jolla, California, Inter-Amer. trop. Tuna Comm. (Final Report for SPOC Contract n? NOO14-72-C-0360).

- 
dy oceanic fronts in the eastern Pacific. La Jolla, California, Inter-Amer. trop. Tuna Comm. (Final Report for SPOC, NOAA Grant n. 04-3-158-59).

- \& - - - 1974b. Comparison of infrared satellite data and fish catch data. Mems Congr. nac. Oceanogr., 5\%, Guaymas, México.

_ KIRKHAM, R. G. 1975. Use of ERTS

(MSS) and NOAA VHRR data in marine resource assessment.
La Jolla, California, Inter-Amer. rrop. Tuna Comm. (Final Report for SPOC NOAA Grant n. 04-5-158-56).

VHRR 3-4 VHRR imagery and LANDSAT multi-spectral scanner images with marine resource measurements. La Jolla, California, Inter-Amer. trov. Tuna Comm. (Final Report for SPOC NOAA Grant n? 04-6-158-44043). 